\title{
Meson-baryon interaction in the meson exchange picture
}

\author{
M. Döring \\ Institut für Kernphysik and Jülich Center for Hadron Physics, \\ Forschungszentrum Jülich, D-52425 Jülich,Germany
}

\begin{abstract}
Elastic $\pi N$ scattering and the reaction $\pi^{+} p \rightarrow K^{+} \Sigma^{+}$are described simultaneously in a unitary coupled-channels approach which respects analyticity. SU(3) flavor symmetry is used to relate the $t$ - and $u$ - channel exchanges that drive the meson-baryon interaction in the different channels. Angular distributions, polarizations, and spin-rotation parameters are compared with available experimental data. The pole structure of the amplitudes is extracted from the analytic continuation.
\end{abstract}

Keywords: Dynamical coupled-channels models, Baryon spectroscopy

PACS: $11.30 . \mathrm{Hv}, 11.80 . \mathrm{Gw}, 13.75 . \mathrm{Gx}, 14.20 . \mathrm{Gk}, 24.10 . \mathrm{Eq}$

\section{INTRODUCTION}

The excitation spectrum of baryons and mesons is expected to reveal important information on the mechanism of confinement as well as the instrinsic structure of hadrons. Properties of baryon resonances have been obtained by lattice calculations [1, 2, 3], mostly for the ground states but also for some excited states [2]. In quark models [4, 5, 6], a rich spectrum of excited states is predicted. Many of these resonances could be identified in elastic $\pi N$ scattering, while at higher energies, usually more states are predicted than seen, a fact commonly referred to as the "missing resonance problem". Since resonances not seen in the $\pi N$ channel might predominantly couple to other channels, there are intensive experimental efforts to measure, among others, multi-pion or $K Y$ final states, where $K Y=K \Lambda$ or $K \Sigma$.

The reaction $\pi^{+} p \rightarrow K^{+} \Sigma^{+}$provides access to a pure isospin $I=3 / 2$ two-body reaction channel in meson-nucleon dynamics. Moreover, the weak decay $\Sigma^{+} \rightarrow p \pi^{0}$ allows to determine the polarization of the produced $\Sigma^{+}$. Thus, we have performed a first combined analysis of the reactions $\pi N \rightarrow \pi N$ and $\pi^{+} p \rightarrow K^{+} \Sigma^{+}$within the unitary dynamical coupled-channels framework [7], with the results summarized in these proceedings.

Dynamical coupled-channels models $[8,9,10,11,12,13,14]$ are particularly suited for combined data analyses: E.g., in the work presented here [7], based on the "Jülich model" [8,9,10, 11], the SU(3) flavor symmetry for the exchange processes allows to relate different final states. The $t$ - and $u$-channel diagrams connect also different partial waves and the respective backgrounds. Thus, the treatment of the interaction via meson and baryon exchange is expected to lead to a realistic background, with strong restrictions on the free parameters. In view of this, the strategy to perform baryon spectroscopy is to introduce only a minimum number of bare resonance states in order to obtain a good description of the data.

The coupled-channels scattering equation $[8,9,10,11]$ used in the present formalism fulfills two-body unitarity, as well as some requirements of three-body unitarity. Furthermore, it fulfills analyticity and takes into account the dispersive parts of the intermediate states as well as the off-shell behavior dictated by the interaction Lagrangians. This integral equation which is solved in the $J L S$-basis is given by

$$
\begin{aligned}
& \left\langle L^{\prime} S^{\prime} k^{\prime}\left|T_{\mu \nu}^{I J}\right| L S k\right\rangle=\left\langle L^{\prime} S^{\prime} k^{\prime}\left|V_{\mu \nu}^{I J}\right| L S k\right\rangle \\
& +\sum_{\gamma L^{\prime \prime} S^{\prime \prime}} \int_{0}^{\infty} k^{\prime \prime 2} d k^{\prime \prime}\left\langle L^{\prime} S^{\prime} k^{\prime}\left|V_{\mu \gamma}^{I J}\right| L^{\prime \prime} S^{\prime \prime} k^{\prime \prime}\right\rangle \frac{1}{z-E_{\gamma}\left(k^{\prime \prime}\right)+i \varepsilon}\left\langle L^{\prime \prime} S^{\prime \prime} k^{\prime \prime}\left|T_{\gamma \nu}^{I J}\right| L S k\right\rangle
\end{aligned}
$$

where $z$ is the scattering energy, $J(L)$ is the total angular (orbital angular) momentum, $S(I)$ is the total spin (isospin), $k\left(k^{\prime}, k^{\prime \prime}\right)$ are the incoming (outgoing, intermediate) momenta, and $\mu, v, \gamma$ are channel indices. In Eq. (1), $E_{\gamma}$ is the on-mass shell energy in channel $\gamma$ [11]. The pseudo-potential $V$ iterated in Eq. (1) is constructed from an effective interaction based on the Lagrangians of Wess and Zumino [15, 16], supplemented by additional terms [9, 10] for including the $\Delta$ isobar, the $\omega, \eta, a_{0}$ meson, and the $\sigma$. The channel space is given by $N \pi, N \eta, N \sigma, \Delta \pi, N \rho[8,9,10]$ and - as the novelty presented here $-\Lambda K$ and $\Sigma K$ [7]. 

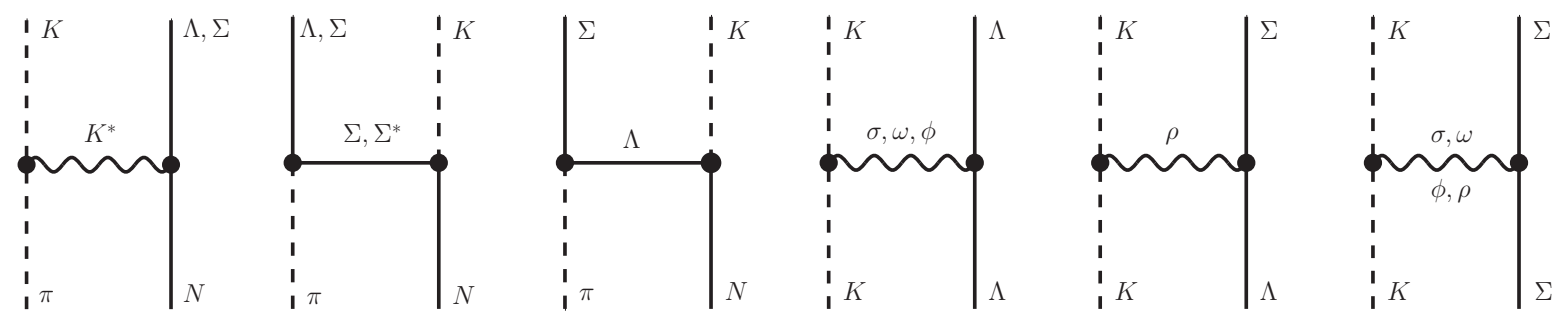

FIGURE 1. $\pi N \rightarrow K Y$ and $K Y \rightarrow K Y$ transitions. For the other transitions used in this approach, see Refs. [9, 10].
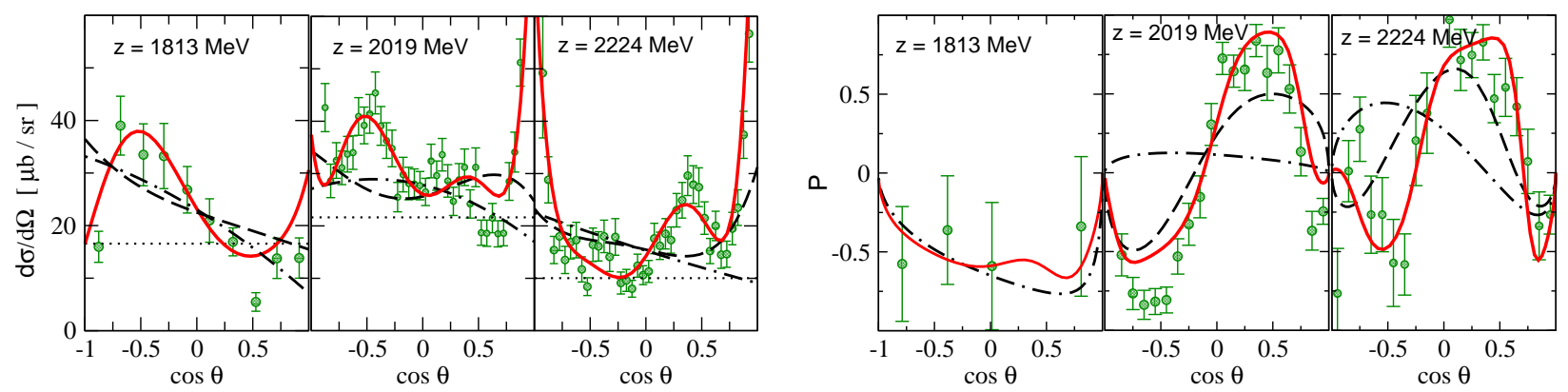

FIGURE 2. Selected results of the presented study (red lines) for three typical energies, for differential cross section and polarization. Data: Ref. [17] and references therein. Also, contributions from different partial waves are shown: Dotted lines: $S$ wave. Dash-dotted lines: $S+P$ waves. Dashed lines: $S+P+D$ waves.

The $t$ - and $u$-channel processes provide the non-resonant interaction in the meson exchange picture. The transition potentials without participation of $K Y$ have been derived in Refs. $[9,10]$ and explicit expressions can be found in these references. In Fig. 1, we show the extension to the $K Y$ channels. Most of the vertices present in these diagrams are related to the ones of Refs. [9, 10] using SU(3) symmetry; details can be found in Ref. [7].

Apart from this non-resonant interaction, $s$-channel diagrams have been introduced, which can be regarded as resonances ( for details, see Ref. [7]. In the present study, we concentrate on the reaction $\pi^{+} p \rightarrow K^{+} \Sigma^{+}$and the isospin $I=3 / 2$ elastic $\pi N$ scattering. Thus, one bare $s$-channel state is included in each of the $I=3 / 2$ partial waves S31, P31, D33, D35, F35, F37. Two are present in P33. These states were allowed to couple to all $I=3 / 2$ channels $\pi N, K \Sigma, \pi \Delta$ and $\rho N$. Together with these four bare couplings, the bare mass has to be left free as a fit parameter. Thus, there are altogether 40 parameters, apart from some free parameters in the $t$ - and $u$-channel diagrams; the latter parameters in the new diagrams from Fig. 1 have been chosen closely to their counterparts without $K Y$ channels from Refs. [10]. Thus, in the present approach there is explicit SU(3) breaking from the different hadron masses, but also a small $\mathrm{SU}(3)$ breaking from the $t$ - and $u$-channel diagrams, mostly in terms of different form factors [7].

\section{RESULTS}

Results for three typical scattering energies $z$ are shown in Fig. 2. The full results for differential cross section $d \sigma / d \Omega$ and polarization $P$, at 32 different energies from the $K \Sigma$ threshold up to $z=2.3 \mathrm{GeV}$, can be found in Ref. [7]. The red solid lines show the result of this study. Overall, the data are well described over the entire energy range. For energies above $2 \mathrm{GeV}$, we do not claim validity of the present model, because the analysis of Refs. [10,11] has been limited to that energy. Consequently, at the highest energies, the $K^{+} \Sigma^{+}$data have not been fitted, but up to $z \sim 2.25$ $\mathrm{GeV}$ the description of the data is still good, as Fig. 2 shows. In Fig. 2, also the influence of individual partial waves is illustrated. At lower energies, $S-P$ wave interference is enough to describe the polarization, but not entirely the differential cross section (dash-dotted lines at $z=1813 \mathrm{MeV}$ ). At higher energies, the figure shows that indeed all partial waves are needed to quantitatively describe the data; in particular, $\mathrm{F}$ waves are important.

Fig. 3 shows the predicted spin-rotation parameter $\beta$ [7] (solid lines). The prediction from the isobar analysis of Ref. [19] is also shown. It is interesting to note that while $d \sigma / d \Omega$ and $P$ are described to a similar precison in both 


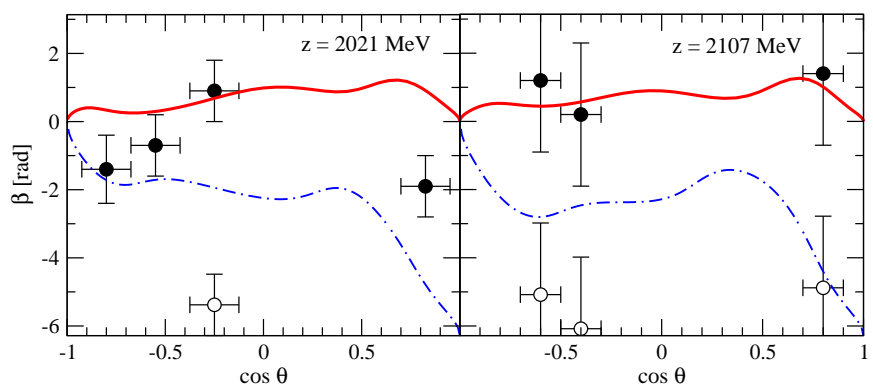

FIGURE 3. Spin rotation parameter $\beta$ of $\pi^{+} p \rightarrow K^{+} \Sigma^{+}$at $z=2021$ and $z=2107 \mathrm{MeV}$. Note that $\beta$ is $2 \pi$ cyclic which leads to additional data points at shifted values shown by the empty circles. Data: Ref. [18]. (Red) solid lines: Prediction from present solution. (Blue) dash-dotted lines: Prediction from Ref. [19].
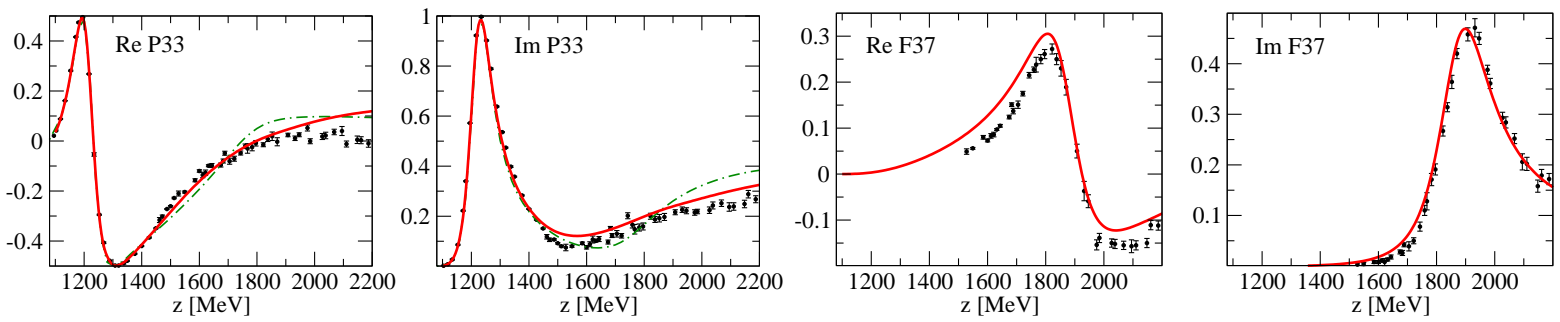

FIGURE 4. Elastic $\pi N \rightarrow \pi N$ partial waves P33 and F37. (Red) solid lines: Present solution. (Green) dash-dotted lines: Jülich model, solution 2002 from Ref. [10]. For the other partial waves see Ref. [7]. Data points: GWU/SAID [20].

studies, the outcome for $\beta$ is so different. This demonstrates that higher precision data for $\beta$ would help further pin down the partial wave content. In any case, what distinguishes the present analysis [7] from Ref. [19] is (apart from conceptual differences) the simultaneous consideration of elastic $\pi N$ scattering. The results of the presented study [7] for the P33 and F37 partial waves are shown in Fig. 4, for the other partial waves see Ref. [7]. The partial waves for the reaction $\pi^{+} p \rightarrow K^{+} \Sigma^{+}$are shown in Ref. [7].

The resonance content of the amplitude is extracted using the analytic continuation following Refs. [11, 21] (see also Refs. [22, 23]). In Table 1, pole positions, residues, and branching ratios for the most prominent resonances in the reaction $\pi^{+} p \rightarrow K^{+} \Sigma^{+}$are shown and compared to results from Refs. [19, 24, 25]. The full list of resonances and their properties can be found in Ref. [7]. As for the well-established 4-star resonances, it is no surprise that most of the pole positions found in this study are in good agreement with the values from the GWU/SAID analysis [20], because

TABLE 1. Left: Resonance pole positions $z_{0}$ in the presented study [7]. Center: $\pi^{+} p \rightarrow$ $K^{+} \Sigma^{+}$residues from Ref. [7]. Right: Transition branching ratios.

\begin{tabular}{cc|c|ccc} 
& $\operatorname{Re} z_{0}[\mathrm{MeV}]$ & $|r|[\mathrm{MeV}]$ & \multicolumn{3}{|c}{$\left(\Gamma_{\pi N}^{1 / 2} \Gamma_{K \Sigma}^{1 / 2}\right) / \Gamma_{\text {tot }}[\%]$} \\
& $-2 \operatorname{Im} z_{0}[\mathrm{MeV}]$ & $\theta\left[{ }^{0}\right]$ & Ref. [7] & Ref. [19] & Refs. [24, 25] \\
\hline$\Delta(1905) F_{35}$ & 1764 & 1.4 & 1.23 & $1.5(3)$ & $<1$ \\
$5 / 2^{+} * * * *$ & 218 & -313 & & & \\
\hline$\Delta(1910) P_{31}$ & 1721 & 5.5 & 2.98 & $<3$ & 1.1 \\
$1 / 2^{+} * * * *$ & 323 & -6 & & & \\
\hline$\Delta(1920) P_{33}$ & 1884 & 5.9 & 5.07 & $5.2(2)$ & $2.1(3)$ \\
$3 / 2^{+* * *}$ & 229 & -38 & & & \\
\hline$\Delta(1930) D_{35}$ & 1865 & 1.6 & 2.14 & $<1.5$ & \\
$5 / 2^{-} * * *$ & 147 & -43 & & & \\
\hline$\Delta(1950) F_{37}$ & 1873 & 2.7 & 2.54 & $5.3(5)$ & - \\
$7 / 2^{+} * * * *$ & 206 & -255 & & & \\
\hline
\end{tabular}


the partial waves from that analysis serve as input for the present study. However, the $\Delta(1920) P_{33}$ and $\Delta(1930) D_{35}$ resonances show no or very small resonance signals in the GWU/SAID analysis of elastic $\pi N$ scattering. Their position is, thus, barely fixed from elastic $\pi N$ scattering. It is then interesting to note that the constraints from the $K^{+} \Sigma^{+}$data lead to resonance positions in vicinity to those quoted in the PDG [26], rated with 3 stars. Thus, we can accumulate further evidence for these states and their positions. Also, we find a $\Delta(1600) P_{33}$ resonance, dynamically generated mainly from the $\pi \Delta$ channel (see also Ref. [27]). Qualitatively, it shares properties with the corresponding state quoted in the PDG [26] like a strong decay into the $\pi \Delta$ channel.

In summary, a first combined analysis of the reactions $\pi N \rightarrow \pi N$ and $\pi^{+} p \rightarrow K^{+} \Sigma^{+}$within the dynamical coupledchannels framework has been presented. In this approach, for both $\pi N$ and $K \Sigma$ a realistic and structured background can be provided. Consequently, only a minimal set of $s$-channel resonances is needed to obtain a good fit to the combined data sets. This is also tied to the fact that in this field-theoretical, Lagrangian based approach, the dispersive parts are fully included and thus, analyticity is ensured. Apart from the well-established 4-star resonances, there is a clear need for the three-star $\Delta(1920) P_{33}$ resonance. This state is found to couple only weakly to $\pi N$ but stronger to $K \Sigma$, and thus evidence for a "missing resonance state" could be accumulated.

\section{ACKNOWLEDGMENTS}

The work of M.D. is supported by DFG (Deutsche Forschungsgemeinschaft, GZ: DO 1302/1-2). This work is supported in part by the Helmholtz Association through funds provided to the virtual institute "Spin and Strong QCD” (VH-VI-231), by the EU-Research Infrastructure Integrating Activity "Study of Strongly Interacting Matter" (HadronPhysics2, grant n. 227431) under the Seventh Framework Program of EU and by the DFG (TR 16). F.H. is grateful to the COSY FFE grant No. 41788390 (COSY-058).

\section{REFERENCES}

1. S. Dürr et al., Science 322, 1224 (2008).

2. J. Bulava et al., Phys. Rev. D 82, 014507 (2010).

3. G. P. Engel, C. B. Lang, M. Limmer, D. Mohler and A. Schäfer [BGR Collaboration], Phys. Rev. D 82, 034505 (2010).

4. N. Isgur and G. Karl, Phys. Rev. D 18, 4187 (1978).

5. S. Capstick and N. Isgur, Phys. Rev. D 34, 2809 (1986).

6. U. Löring, B. C. Metsch and H. R. Petry, Eur. Phys. J. A 10, 395 (2001).

7. M. Döring, C. Hanhart, F. Huang, S. Krewald, U.-G. Meißner and D. Rönchen, arXiv:1009.3781 [nucl-th].

8. C. Schütz, J. Haidenbauer, J. Speth and J. W. Durso, Phys. Rev. C 57, 1464 (1998).

9. O. Krehl, C. Hanhart, S. Krewald and J. Speth, Phys. Rev. C 62, 025207 (2000).

10. A. M. Gasparyan, J. Haidenbauer, C. Hanhart and J. Speth, Phys. Rev. C 68, 045207 (2003).

11. M. Döring, C. Hanhart, F. Huang, S. Krewald and U.-G. Meißner, Nucl. Phys. A 829, 170 (2009).

12. B. Juliá Díaz, T. S. Lee, A. Matsuyama and T. Sato, Phys. Rev. C 76, 065201 (2007).

13. N. Suzuki, B. Juliá Díaz, H. Kamano, T. S. Lee, A. Matsuyama and T. Sato, Phys. Rev. Lett. 104, 042302 (2010).

14. L. Tiator, S. S. Kamalov, S. Ceci, G. Y. Chen, D. Drechsel, A. Svarc and S. N. Yang, arXiv:1007.2126 [nucl-th].

15. J. Wess and B. Zumino, Phys. Rev. 163, 1727 (1967).

16. U.-G. Meißner, Phys. Rept. 161, 213 (1988).

17. D. J. Candlin et al., Nucl. Phys. B 226, 1 (1983).

18. D. J. Candlin et al., Nucl. Phys. B 311, 613 (1989).

19. D. J. Candlin et al. [Edinburgh-Rutherford-Westfield Collaboration], Nucl. Phys. B 238, 477 (1984).

20. R. A. Arndt, W. J. Briscoe, I. I. Strakovsky and R. L. Workman, Phys. Rev. C 74, 045205 (2006).

21. M. Döring, C. Hanhart, F. Huang, S. Krewald and U.-G. Meißner, Phys. Lett. B 681, 26 (2009).

22. M. Döring and K. Nakayama, Eur. Phys. J. A 43, 83 (2010).

23. M. Döring, D. Jido and E. Oset, Eur. Phys. J. A 45, 319 (2010).

24. G. Penner and U. Mosel, Phys. Rev. C 66, 055211 (2002).

25. V. Shklyar, G. Penner and U. Mosel, Eur. Phys. J. A 21, 445 (2004).

26. K. Nakamura et al., Journal of Physics G 37, 075021 (2010).

27. E. Oset and A. Ramos, Eur. Phys. J. A 44, 445 (2010). 